\title{
Detection of Parkinson Diseases With More Accuracy using Machine Learning Technique
}

\author{
P. Sai Dinkar, M.K.Mariam Bee
}

\begin{abstract}
Parkinson's malady is the most common neurodegenerative confusion influencing more than 10 million individuals around the world. There is no single test which can be regulated for diagnosing Parkinson's illness. In light of these challenges, to explore a machine learning way to deal with precisely analyze Parkinson's, utilizing a given dataset. To keep this issue in medicinal parts need to anticipate the malady influenced or not by discovering exactness figuring utilizing $A I$ strategies. The point is to examine AI based methods for Parkinson sickness by expectation results in best precision with finding arrangement reportIn the beginning times of Parkinson ailment, your face may appear practically zero articulation. Your arms may not swing when you walk.. At times, your specialist may recommend medical procedure to manage certain locales of your cerebrum and improve your indications.To propose, an AI based strategy to precisely foresee the illness by discourse and tremor manifestations by expectation results as best exactness from looking at administer grouping AI calculations. Also, to look at furthermore, talk about the execution of different AI calculations from the given transport traffic division dataset with assessment arrangement report, distinguish the outcome demonstrates that the viability of the proposed AI calculation procedure can be thought about with best exactness with accuracy, Recall and F1 Score.
\end{abstract}

Keywords-dataset, machine learning classification method, python.

\section{INTRODUCTION}

Machine learning predictAI isto foresee the longer term from past info. AI (ML) could be a reasonably computerised reasoning (AI) that furnishes PCs with the capability to find out while not being without ambiguity customised. AI centers round the improvement of laptop comes which will amendment once given to new info and also the necessities of Machine Learning, execution of a basic AI calculation utilizing python. Procedure of preparing and expectation includes utilization of particular calculations. It feed the preparation information to a calculation,also, the calculation utilizes this preparation information to give expectations on another test information. Machine learning can be generally isolated in to three classifications. There are regulated learning, unsupervised learning and fortification learning. Directed learning program is both given the info information and the relating marking to learn information must be named by an individual already. Unsupervised learning is no

Revised Manuscript Received on September 10, 2019.

P. Sai Dinkar, Saveetha school of Engineering(SIMATS) Department of Electronics And Communication, Perambakkam, Tamilnadu, India.

(Email: saidhinakarchowdary@gmail.com)

M.K.Mariam Bee, Saveetha school of Engineering(SIMATS) Department of Electronics And Communication, Perambakkam, Tamilnadu, India.

(Email: mariamresearch09@gmail.com) names. It gave to the learning calculation. This calculation needs to make sense of the grouping of the information. At long last, Reinforcement learning powerfully collaborates with its condition and it gets positive or negative input to Parkinson's infection a long haul degenerative confusion of the focal sensory system that influences the engine control of a patient by influencing predominately dopamine delivering ("dopaminergic") neurons in a particular territory of the cerebrum. The principle issue in recognizing the illness auspicious is the noticeable side effects show up for the most part at the later stage where fix never again ends up conceivable. There is no right reason demonstrated yet that outcomes to reason for Parkinson's, thus researchers are as yet leading broad research to discover its accurate reason. In spite of the fact that some anomalous qualities that become noticeable because of older seem to prompt Parkinson's in a few people however there is no proof to evidence this. In spite of the fact that there are two or three systems for early Parkinson's location. Dopamine transporter single-photon discharge processed tomography can be utilized to viably conclusion Parkinson's by recognizing measure of dopamine inadequacy in the concerned patient's mind cell at an impressive beginning time.From all subjects, three kinds of penmanship accounts (Static Spiral Test (SST), Dynamic Spiral Test (DST) and Stability Test on Certain Point (STCP)) are taken.. Likewise the illustrations of spirals has a place with the PWP are incorporated into the dataset. Discourse dataset, The Multi-Dimensional Voice Program (MDVP) is an entrenched programming Program utilized for quantitative acoustic flag evaluation of voice quality. The MDVP figures various acoustic parameters including gleam, present moment annoyances of the abundancy, and jitter, transient irritations of the recurrence. Bound together Parkinson\&\#39;s malady Rating Scale(UPDRS) report the rationale for this check is to determine the modification in patient\&; drawing execution what is additional, delay times since it's more and more arduous to follow the Archimedean winding during this case. attributable to this check, it's seen that the bigger a part of the patients unbroken illustration be that because it might, concerning each one of them lost the instance. The third check is Stability check on bound purpose (STCP). during this check, there's a certain red purpose amidst the screen and also the subjects square measure requested to carry the advanced pen on the purpose while not contacting the screen during a bound time. The motivation behind this check is to determine the 
patient;hand strength or hand tremor level.Procedures of Directed Machine Learning calculations incorporate strategic relapse, multi-class grouping, call Trees and bolster vector machines then forth. Regulated learning requiresAIPastDataset that the knowledge accustomed prepare the calculation is as of currently named with right answers. Managed learning problems are often in addition gathered into Classification problems. This issue has as objective the event of a aphoristic model that may anticipate the estimation of the indigent attribute from the standard factors. the excellence between the 2 assignments is that the manner that the subordinate quality is numerical for downright for order.An order demonstrateendeavors to make some inference from watched esteems. Given at least one data sources a characterization model will endeavor to anticipate the estimation of at least one results.

\section{WORKING PRINCIPLE}

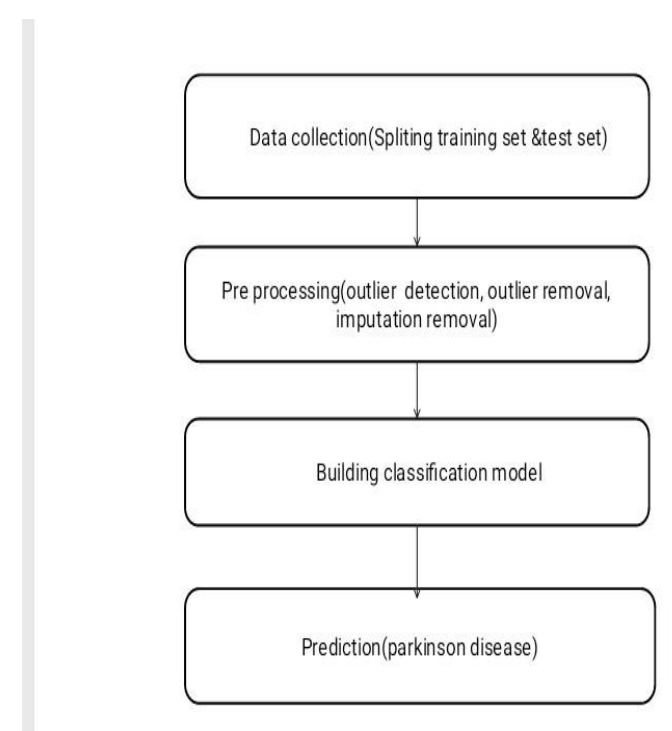

Fig1: data flow diagram for Machine learning model

\section{Data Collection:}

The informational index gathered for foreseeing advance shoppers is a component into coaching set and check set. For the foremost half, 7:3 proportions area unit connected to half the coaching set and check set. the info Model that was created utilizing Random Forest, calculated, call tree calculations and then forth area unit connected on the coaching set and enthusiastic about the check outcome preciseness, check set forecast is finished.

\section{Preprocessing}

The information which was gathered may contain missing qualities that may prompt irregularity. To increase better outcomes information should be preprocessed in order to improve the effectiveness of the calculation. The anomalies must be evacuated and furthermore factor change should be finished. In light of the connection among qualities it was seen that properties that are huge independently.

\section{Building classification model:}

It is study in preprocessing outliers, orthogonal variables,anda mixture of continuous,categorical and separate variables.

\section{EXISTINGSYSTEM}

To exhibit the plausibility of assessing AI calculations to group

PD subjects from HC. The settled cross-approval utilized in this work was a basic segment to choose solid models. The SVM and MLP models somewhat outflank the different models utilizing engine includes as was the K-NN utilizing non-engine highlights. The held models were connected on SWEDD subjects to foresee cost-delicate learning. The impediment with under examining is that it disposes ofconceivably valuable information. The principle hindrance with oversampling, from our point of view, is that by making precise of existing precedents, it makes over fitting likely.truth be told, with oversampling it's terribly traditional for a student to form AN order principle to hide a solitary, duplicated, precedent. A second impairment of oversampling is that it expands the number of getting ready precedents, consequently increasing the training time.The MLP show gave AN order of SWEDD as pursue: a forty seventh of SWEDD patients were delegated metallic element engine issue twins by the MPL_M show and forty first were named having a begin non-engine irregularity of metallic element by MPL_NM show. Cross the 2 models, twenty sixth of SWEDD patients were named having each metallic element highlights. The LR show gave a characterization of as pursue: a forty seventh of SWEDD patients were delegated metallic element engine issue carbon copies by the primary LR_M demonstrate and thirty eighth were delegated having a beginning non-engine variation from the norm of metallic element endlessly show. Cross the 2 models, pure gold of SWEDD patients were named having each metallic element highlights. There area unit completed inconveniences connected with the employment of inspecting to execute cost-delicate learning. The impediment with underneath examining is that it disposes of conceivably valuable info. The principle hindrance with oversampling, from our purpose of read, is that by creating precise of existing precedents, it makes over fitting truth be told, with oversampling it's terribly traditional for a student to form AN order principle to hide a solitary, duplicated, precedent. A second impairment of oversampling is that it expands the number of getting ready precedents, consequently increasing the training time.

\section{DICUSSION}

DatSCAN Single-photon emanation processed imaging (SPECT) imaging could be a solid technique to survey Dopaminergic Transporter in chronic Parkinson's syndrome. Output while not proof of dopaminergic inadequacy (SWEDD) area unit subjects clinically analyzed as encephalopathy illness (PD) patients in spite of the actual fact that the SPECT imaging doesn't demonstrate any negrostriatal variation from the norm. To five models of AI were utilised to try and do double characterization (solid control/PD) utilizing clinical appraisal and movie inferred highlights connected from that time on SWEDD amass as a possible utilization of engine and non-engines includes in 
understanding Parkinson infection trademark during this gathering. The settled cross-approval was a elementary section to decide on dependable models.

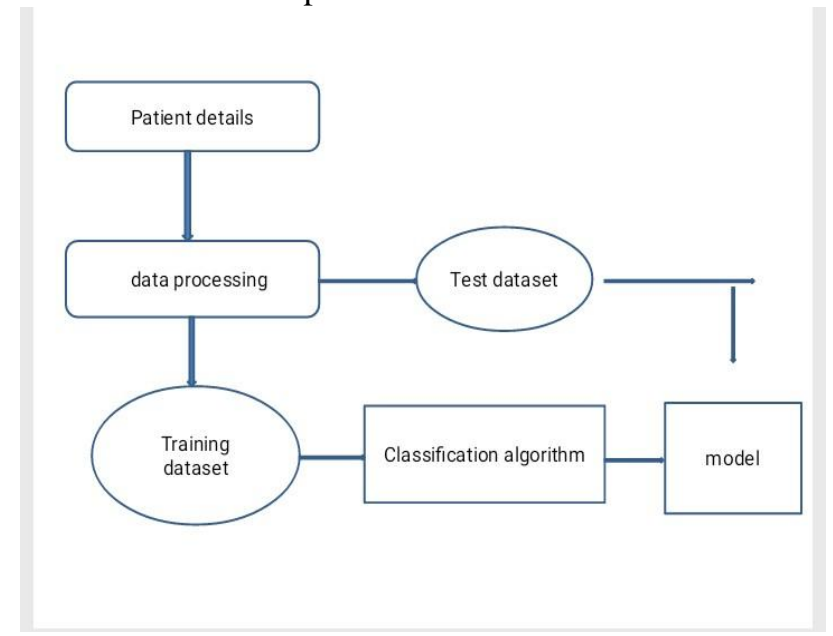

FIG 2:Architecture of proposed block
Environmental Requirements:

1.Software Requirements:

Operating System: Windows / Linux

Simulation Tool: Anaconda with Jupyter Notebook

2. Hardware requirements:

Processor: Pentium IV/III

Hard disk: minimum $80 \mathrm{~GB}$

RAM: minimum 2 GB

\section{TEST \& RESULT}

Unnamed: 0 - 1 0.130.220.240.25-0.3-0.340.230.350.380.290.190.270.130.260.340.190.160.230.110.19-0.3-0.180320.270.180.370.220.120.320.69

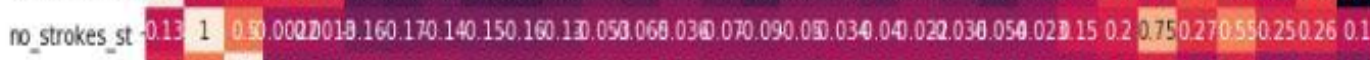

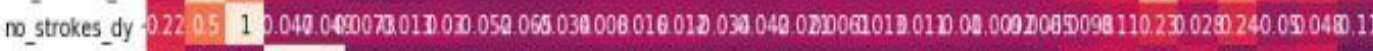

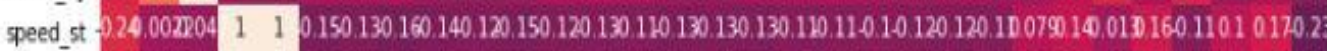

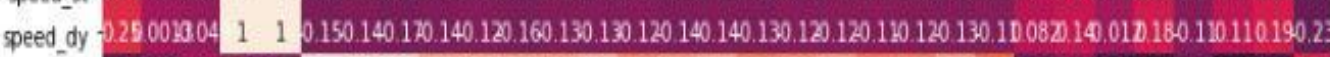
magnitude vel st $-0.30 .15007 .150 .191 \quad 0.970 .960 .780 .780 .750 .730 .820 .620 .680 .761580 .630 .70550570710490 .240 .110 .380 .440 .230 .490 .390 .22$. nagnitude_horz vel_st 0.340 .170 .018 .130 .10 .9710 .880770 .810 .687610 .770470570720444050 .63040430 .620340 .10 .089 .370 .430 .270 .480 .39023

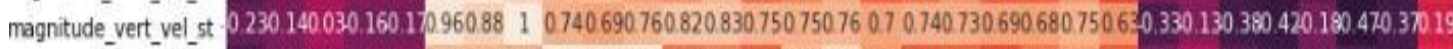

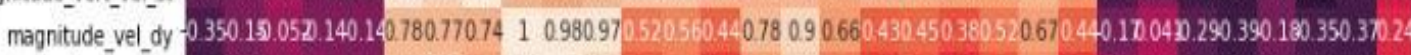

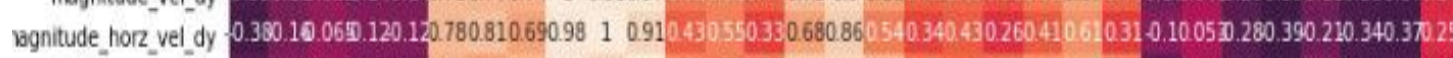

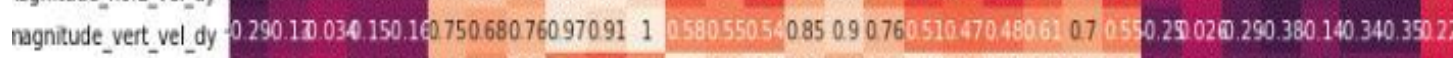

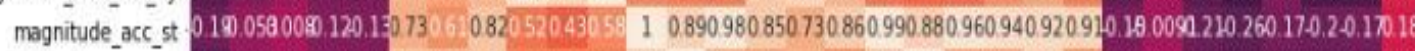

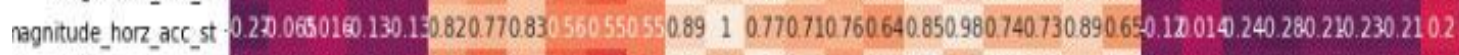

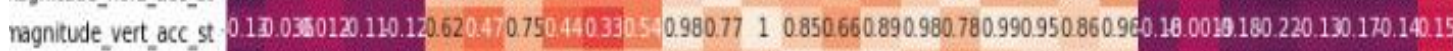
magnitude_acc_dy $\quad 0.260 .070 .030 .130 .140 .6805770 .750 .780 .680 .850 .850 .710 .85 \quad 1 \quad 0.930 .980 .830 .680 .820 .920 .910800 .1970420 .210 .280180 .220 .2021$ lagnitude_horz_acc_dy 0.340 .07040130 .140 .760 .720 .7609086090 .730 .760660931083068070620750890670 .120090 .250310 .230 .260 .240 .26

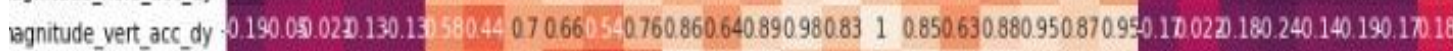

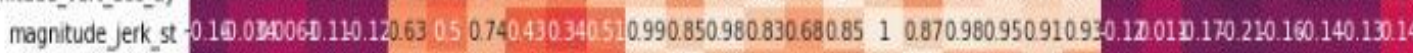
agnitude_horz jerk_st $0.230 .00010 .110 .120 .70 .630730450430470 .880 .980780 .680 .70630 .87 \quad 1 \quad 0.770 .750 .8906500900290 .180 .230 .100 .160 .15015$

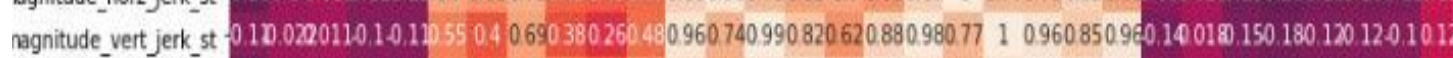

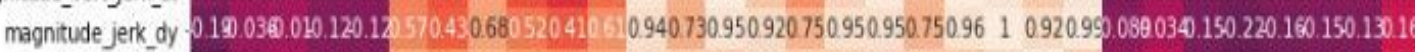

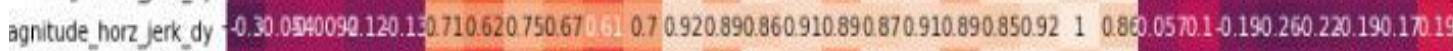

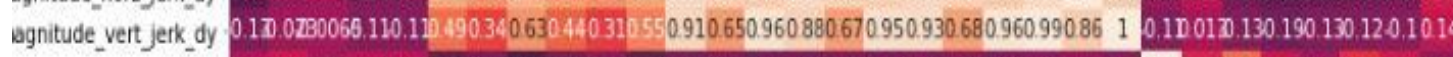

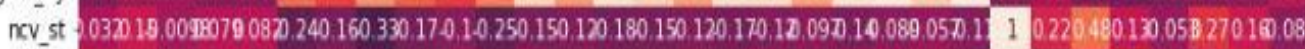

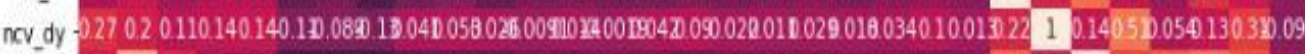

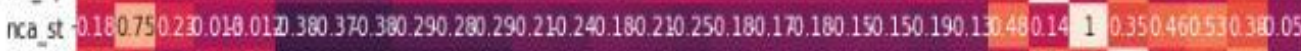

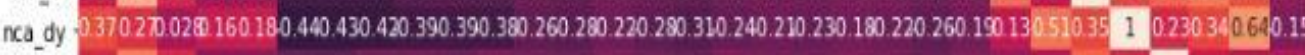

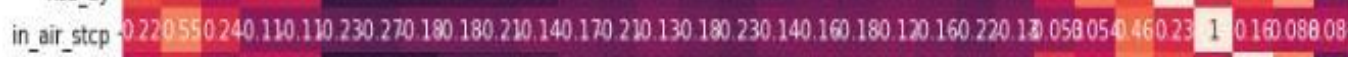
on_surface_st $-0.120250 .050 .10 .110 .490 .480 .470 .350 .340 .340 .2-0.230 .170 .220 .260 .190 .140 .160 .120 .150 .190 .120 .270 .130530340 .1610550 .26$

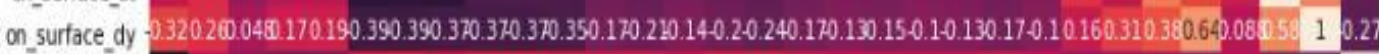
target 0.690 .10 .110 .230 .230 .220 .230 .190 .240 .250 .220 .180 .20 .150 .210 .240180 .140 .150 .120 .160 .190 .10 .080 .093 .050 .10080 .260 .271

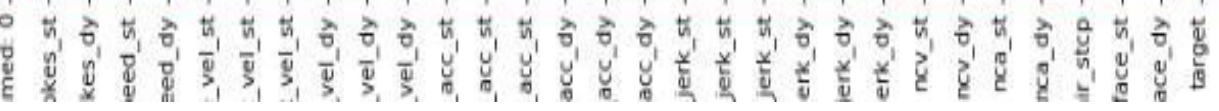

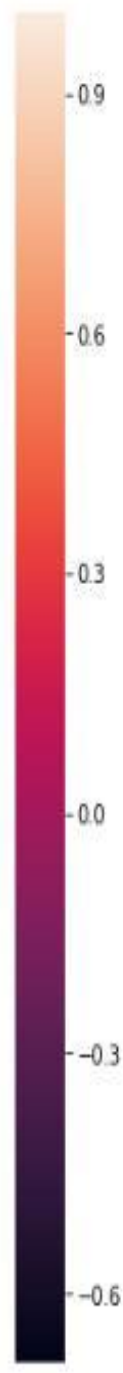

Fig 3: Heat map diagram of given dataset for tremor

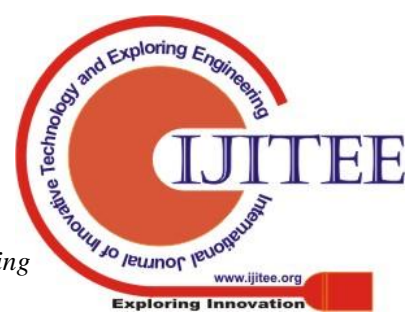




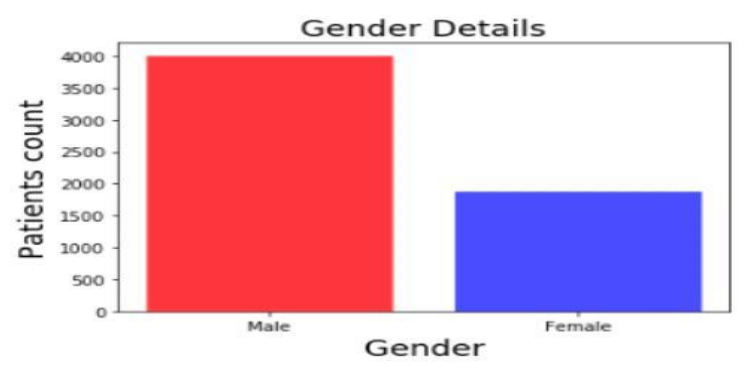

Fig: Gender details of given dataset

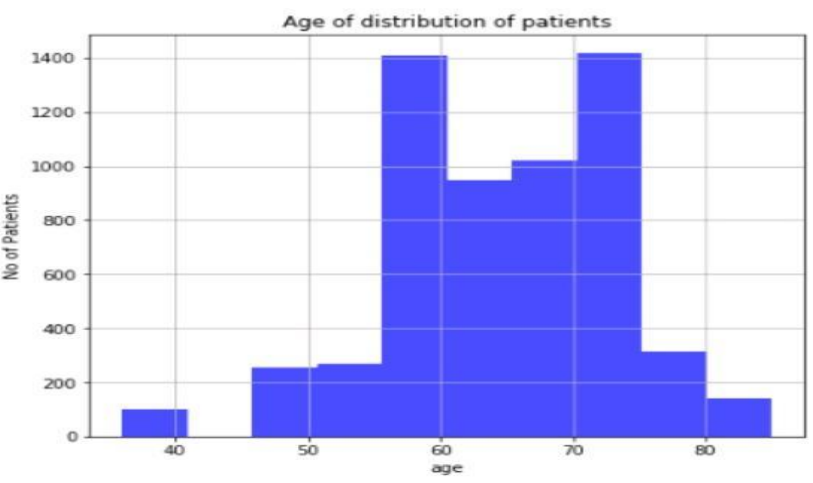

Fig: Age distribution of each patients

Fig 4:Gender Details\&Age Distribution Of Each Patients

\section{PROS AND CONS}

A patient's stay at a clinic may include different divisions or units. Since numerous basic and complex issues happen at the interfaces of social insurance conveyance frameworks like discovery To sheltered and effective changes between the divisions inside a clinic has noteworthy significance for machine learningTo spare specialists time. We can separate people dependent on weight with dataset. Informational collection like controlled individual, recognizing individual.

\section{CONCLUSION}

We propose this investigation to exhibit the attainability of assessing AI calculations to rearrange metal subjects from HC. The settled cross-approval used during this work was anbasic half to settle on dependable models. MLP models somewhat beat alternate models utilizing engine includes as was the K-NN utilizing non-engine highlights. The control models were connected on SWEDD subjects to foresee their conditions as a metal understanding with engine issue or as a metal tolerant with non-engine variation from the norm. The means that the ground- truth of this gathering is inaccessible makes the assessment the execution of those models on this gathering cumbersome.

\section{REFERENCES}

1. Kovacs, G.G.: 'Concepts and classification of neurodegenerative diseases', Handbook of clinical neurology, 2017, 145, pp. 301-307

2. Molteni, M., and Rossetti, C.: 'Neurodegenerative diseases: The immunological perspective', Journal of neuroimmunology, 2017, 313, pp. 109-115

3. Griesbach, G.S., Masel, B.E., Helvie, R.E., and Ashley, M.J.: 'The Impact of Traumatic Brain Injury on Later Life: Effects on Normal Aging and Neurodegenerative Diseases', Journal of neurotrauma, 2017
4. El Kadmiri, N., Said, N., Slassi, I., El Moutawakil, B., andNadifi, S.: 'Biomarkers for Alzheimer Disease: Classical and Novel Candidates' Review', Neuroscience, 2017

5. Pohanka, M.: 'Alzheimer s disease and oxidative stress: a review', Current medicinal chemistry, 2014, 21, (3), pp. 356- 364

6. Yau, Y., Zeighami, Y., Baker, T.E., Larcher, K., Vainik, U., Dadar, M., Fonov, V.S., Hagmann, P., Griffa, A. Misic, B., Collins, D.L., and Dagher, A.: 'Network connectivity determines cortical thinning in early Parkinson's disease progression', Nature communications, 2018, 9, (1), pp. 12

7. Apfeld, J., and Fontana, W.: 'Age-Dependence and Aging-Dependence: Neuronal Loss and Lifespan in a C. elegans Model of Parkinson's Disease', Biology, 2017, 7, (1)

8. Londos, E.: 'Practical Treatment of Lewy Body Disease in the Clinic: Patient and Physician Perspectives', Neurology and therapy, 2017. 